\title{
Clinical and experimental medicine: innovation and continuity
}

\author{
Franco Dammacco
}

Published online: 14 February 2012

(C) Springer-Verlag 2012

In the last few years, Clinical and Experimental Medicine (CEM) has recorded a slow but steady growth in the scientific soundness of the contributions accepted for publication, and consequently in its impact factor and the size of its readership. In parallel, there has been a progressive increase in the subscription preference for the "online only" rather than the "print + online" modality. Thus, in recognition of this shift by the international scientific community and the ecological implications of any such decision, starting from its first issue of 2012, CEM will be published exclusively online.

This editorial change is also an opportunity to reaffirm the continuity but also the diversity of CEM by announcing that the journal's board has been extensively renewed. On this occasion, I would like to express my deep gratitude for the support provided by the colleagues who have long served on the editorial board and are now stepping down. At the same time, a hearty welcome is addressed to the leading international scholars in selected fields of basic and clinical medicine who have kindly accepted my invitation to join the new international editorial board of CEM. These scientists offer the benefits of their specific expertise and join the six editors retained from the previous board, whose continuing efforts will be well appreciated. I am confident that, with the help of this distinguished group, the peer-review system of the journal will be substantially strengthened.

Further changes will affect the overall structure of CEM. Obviously, its mission to attract and assess innovative research of basic as well as clinical interest, produced worldwide by highly capable investigators, remains

F. Dammacco $(\bowtie)$

Editor-in-Chief

Bari, Italy

e-mail: francodam@dimo.uniba.it unchanged. However, CEM will more formally narrow its scientific interest to hematology, onco-hematology, oncology, virology, immunology, and rheumatology, as these are the fields indeed represented by the majority of the manuscripts submitted to the journal.

Along with the more traditional types of articles, namely reviews, regular papers on experimental and pre-clinical studies, and translational research, the journal now wishes to encourage the submission of prospectively designed clinical trials and epidemiological studies. Furthermore, it will publish, although with no pre-defined frequency, invited editorial commentaries that are meant to provide an in-depth discussion highlighting exciting research published in the same issue. The Editor-in-Chief, the Associate Editors, and members of the Editorial Board, depending on their specific expertise, will be largely responsible for the timely preparation of such commentaries, which will focus on a few essential points, such as the relevance of the research and its implications in the context of current information, the strengths and weaknesses of the work, and future perspectives. In addition, expert investigators will be invited to share their insight and experience into the fields of research covered in a particular issue, based on a confidential "sneak preview" of the manuscript(s). The length of these commentaries should not exceed 1,500 words and should include no more than 1 table/figure and 10 references.

The overall management of CEM will also undergo a few changes. Readers of primary-source periodicals deserve to be confident that what they are reading is original; thus, following the online submission of a manuscript via Editorial Manager ${ }^{\circledR}$, any ethical misconduct will be seriously dealt with, and plagiarized manuscripts will obviously and immediately be rejected. Articles will be peer-reviewed and every effort will be made to ensure that the author receives the Editor's decision together with 
Reviewers' comments in a timely fashion, hopefully within 2 months of submission. In order to achieve this goal, the manuscript will undergo a two-stage process: (a) assignment to an appropriate member of the Editorial Board, who will decide within a matter of days whether the manuscript should be sent for peer review; (b) if the decision is yes, the manuscript will be assessed by two or in some cases three independent reviewers. A reasonable estimate of the average duration of stage two is $30-40$ days. The final decision, left to my responsibility, will then be e-mailed to the corresponding author.

Accepted articles will be published in one of the next two issues of the journal, thereby resulting in immediate international exposure with Pubmed/MEDLINE indexation. Correction via e-mail of electronically retrieved page proofs by the corresponding author within three working days will further speed up the reviewing process and ultimately result in the appearance of the article on EarlyView, until it is assigned to an issue. Free PDF/digital files are granted to the authors of published papers. Thus, by combining high-quality research with an accelerated editorial review process and innovative technology, faster and more efficient publication is expected, with the final desirable aim of the further cultural growth of CEM and its transformation from a quarterly to a bimonthly journal. 